\title{
Fermi-Pasta-Ulam recurrence and coupled discrete Ginzburg-Landau chains in the description of various in vitro states of cell differentiation in the blood of healthy donors and acute-leukemia patients
}

\author{
Berezin AA* \\ EC-leasing Moscow, Russia
}

\begin{abstract}
The subject of the manuscript is the development of a fundamental physical model for differentiation of healthy blood cells to leukemic ones. Their main result is that the approach utilizing the Ginzburg--Landau chain and the Fermi-Pasta-Ulam (FPU) recurrence concept helps to describe the mechanism of transition of healthy blood cells into leukemic ones. The development of leukemia in the framework of the model is described as (1) a decrease of the attractor dimension in the model of the differentiation in blood cells (described by a Ginzburg--Landau chain) and (2) a transition from the cycle of complex attractors in the sequence of basic cell differentiation states into the cycle of simple attractors or simple FPU recurrences of leukemic (tumor) cell differentiation.
\end{abstract}

\section{Introduction}

Some researches assume that initiation of tumor processes is connected with disruption of cell differentiation [1]. This process is often considered as a cell specialization: a differentiated cell has got some functions common for many cells. So the problem of differentiation in reality represents a problem a different genes activity $[2,3]$. However, the mechanisms of ones genes activation and suppression of the others are not completely known.

In connection with this interpretation of physical mechanism of one of the most demonstrative tumor processes - acute leukemia, the origin of leukemia cells from healthy blood cells requires a fundamental approach within the framework of dynamical systems. Found in vitro conditions oscillations of absolute concentrations of normal (healthy) and tumor (leukemic cells) [4] allowed to put forward a hypothesis of the physical mechanism of cell differentiation.

\section{Materials and methods}

$200 \mathrm{ml}$ blood probes of 20 healthy donors and of 20 patients with acute lymphoblastic leukemia were kept in a thermostat for 10 hours. Every 15 minutes the blood from the probes was taken for analysis of concentrations of normal (healthy) and tumor (leukemic cells) in 1 cubic mm [4]. Then the point meanings of concentrations were approximated by oscillatory functions. The typical curves of the concentrations of oscillations of polymorphonuclear neutrophils, lymphocytes, and tumor (leukemic) are presented in Figure 1 and 2.

\section{Mathematical model of cell differentiation}

A previous paper by our group [4] suggested a mathematical model of concentration oscillations of polymorphonuclear neutrophils, lymphocytes, and tumor (leukemic) cells in the in vitro the blood of healthy donors and acute-leukemia patients. This model allowed one to describe experimental data. At the same time, the interpretation of the physical mechanism of the leukemia development from healthy blood cells calls for a fundamental approach based on dynamical systems. In an effort to develop a fundamental physical model for differentiation of healthy blood cells to leukemic ones, we considered the following hypothesis. Gaponov, et al. [5] showed that a nonlinear interaction

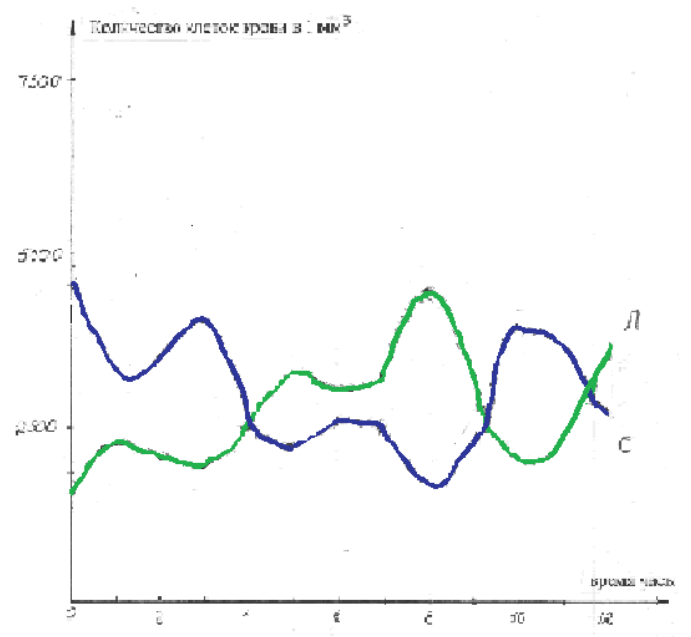

Figure 1. Typical curves of the concentrations oscillations of polymorpho nuclear neutrophils and lymphocytes in the blood of healthy donors. The ordinate is the cell concentration.

Correspondence to: Berezin AA, EC-leasing Moscow, Russia, artparis@mail.ru

Key words: Fermi-Pasta-Ulam recurrence, Ginzburg-Landau chain, cell differentiation, in vitro oscillations of blood cell concentrations

Received: March 04, 2017; Accepted: April 07, 2017; Published: April 10, 2017 


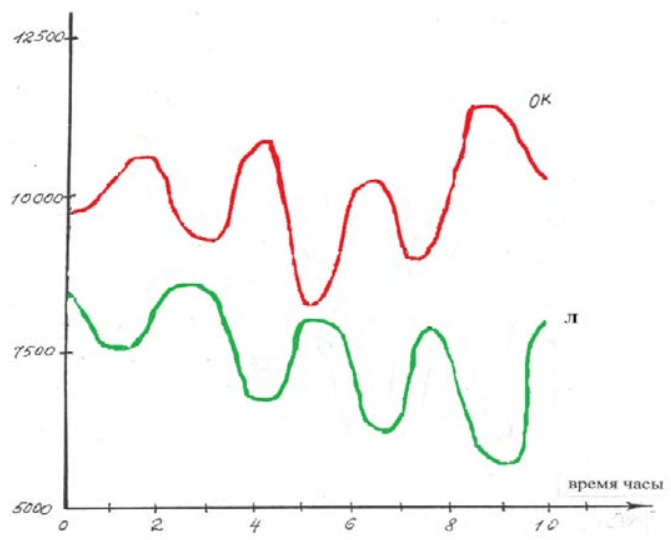

Figure 2. Typical curves of the concentrations oscillations of lymphocytes and tumor (leukemic) in the blood of patients with acute lymphoblastic leukemia. The ordinate is the cell concentration.

of the collective and the local oscillations in a chain of sequentially coupled self-excited Van der Pol type oscillators (described by a discrete chain of the Ginzburg--Landau equations) may result in the establishment of stochastic self-oscillations in the system. A transform of these oscillations is a strange attractor. That corresponds to the complex FPU recurrence [6] which represents a return the picture of the spectrum the system perturbations to its original form. On the other hand, Yudovich [7] showed that the Van der Pol equation with a time lag can by a change of variables be reduced to the Lorentz system [8]. The given above allows one to consider the Ginzburg--Landau chain as a chain of coupled attractors in the space of time lags and eigenfrequences of the self-excited oscillators of the chain. Assume that various types of blood cells are represented as a dynamical sequence of transitions between different attractors or FPU recurrences in the space of blood cell differentiation times $T_{x}, T_{y}, T_{z}, T_{k}$; the eigenfrequencies $\omega_{1}, \omega_{2}$, of the cell concentration oscillations; and the frequencies $\omega_{3}, \omega_{4}$ of mechanical oscillations in a DNA molecule in the framework of the discrete Ginzburg-Landau chain [2] subjected to an external noise simulating a thermal effect of a surrounding solution on the cells. In this case, the model of differential activity of genes is reduced to maintaining the corresponding number of the blood cell differentiation states observed in experiment; that is, to a certain dimension of the attractor in the dynamical system. In addition, there exist both the direct and inverse blood cell differentiation cycles. The scheme of these cycles is as follows. In the direct cycle, a polymorphonuclear neutrophil differentiates to a band neutrophil and then to a small lymphocyte. The latter again differentiates to the polymorphonuclear neutrophil via the stage of band neutrophil. The inverse cycle proceeds somewhat differently. A lymphocyte differentiates to a monocyte and then to a polymorphonuclear neutrophil. The latter again differentiates to the lymphocyte via the stage of monocyte. These sequences of blood cell differentiation were experimentally obtained by Makarov [9]. The direct and inverse cycles of blood cell differentiation were described in our model with the aid of two parametrically coupled GinzburgLandau chains consisting of five sequentially coupled self-excited Van der Pol type oscillators (according to the number of experimentally observed blood cell differentiation states)

$$
\begin{aligned}
& \frac{d a_{j}}{d t}=a_{j}\left(1-\delta\left|a_{j}\right|^{2}\right)+\gamma\left(a_{j}-a_{j-1}\right)\left(b_{j}-b_{j-1}\right)+c_{1} F_{1}+c_{2} F_{2} \\
& \frac{d b_{j}}{d t}=b_{j}\left(1-\delta\left|b_{j}\right|^{2}\right)+\gamma\left(b_{j}-b_{j-1}\right)\left(a_{j}-a_{j-1}\right)+c_{1} F_{1}+c_{2} F_{2}
\end{aligned}
$$

where $a_{j}, b_{j}$ are Fourier spectra corresponding to various states of differentiation of polymorphonuclear neutrophils and lymphocytes, respectively; $\omega_{1}, \omega_{2}$, are initial frequencies of the blood cell concentration oscillations (these frequencies correspond to the initial differentiation states in the first and second cycles); $j=1,2, \ldots 5$ is the number of differentiation states in the first and the second cycles; and $\delta, \gamma$ are coefficients of nonlinearity and of parametric interaction of the cycles, respectively. The function $F_{1}$ describes a Poisson random process simulating stochastic oscillations in blood plasma proteins; the probability that one or several jumps of the concentration will occur within the time interval $(\mathrm{t}, \mathrm{t}+\mathrm{h})$ is

$$
P_{j}(h)=1-\exp (-\lambda h)
$$

Here $\lambda$ is a constant and the function $F_{2}$ describes a Gaussian random process (with a probability density $\mathrm{p}(\mathrm{y})$ ) simulating thermal fluctuations of protein mobility in the solution

$$
p(y)=\frac{1}{\sqrt{2 \pi} \sigma} \exp \left(-\frac{(y-\mu)^{2}}{2 \sigma^{2}}\right)
$$

where $\mu$ and $\sigma^{2}$ are the averaged value and the variance of the quantity y. The functions $F_{1}$ and $F_{2}$ were realized with the aid of standard program operators. The coefficients $c_{1}$ and $F_{1}$ were varied from 0 to 0.1 . The $F_{1}$ and $F_{2}$ functions are taken to be independent of $a_{j}, b_{j}$. A numerical study of system (1) showed that a beating chaos mode (or a complex Fermi--Pasta--Ulam (FPU) recurrence) is stabilized in such a chain in a wide range of frequencies $\omega_{1}, \omega_{2}$ (from $\frac{\omega_{2}}{\omega_{1}}=2$ to $\frac{\omega_{2}}{\omega_{1}}$ $=10)$ and amplitudes of the external random forces $F_{1}, F_{2}\left(c_{1}\right.$ and $c_{2}$ were varied from 0 to 0.4 ). Comparison of the initial (a and b) and final (e and $\mathrm{f}$ ) cycles in the model of in vitro blood cell differentiation suggests a conclusion that the mode of reversible transition between the attractors (or different Fourier spectra) takes place in the dynamics of both chains (Figure 3-6).

Figure 5 shows the computer-simulated dynamics of beating between the Fourier spectra reflecting the differentiation states of polymorphonuclear neutrophils (PN) and lymphocytes (L) under in vitro conditions. As is evident from the plots in Figure 5, the phases of the oscillations are stable and exhibit practically no drift (just as in experiment [4]). If the number of the chain equations is diminished by one, the simulation of beating between the Fourier spectra (Figure 6) reflecting the differentiation states of lymphocytes (L) and tumor cells (TC) in vitro shows a drift of the concentration oscillation phases (Figure 6) (in agreement with the experimental data [4]).

\section{Discussion}

As a result of computer study of the model it was shown that the model curves (Figure 5 and 6) proved to be close to experimental ones (Figure 1 and 2).

Thus, the simulation of beating between the Fourier spectra reflecting the differentiation states of (1) polymorphonuclear neutrophils and lymphocytes in the blood of healthy donors and (2) lymphocytes and tumor cells in the blood of acute-leukemia patients under in vitro conditions in the framework of the dynamics of coupled discrete Ginzburg--Landau chains showed that different blood cell differentiation states can be interpreted as attractors in the space of durations of cell differentiation process and eigenfrequencies of the blood cell concentration oscillations and the mechanical oscillations in the DNA molecule (between which the reversible transition or 
a)

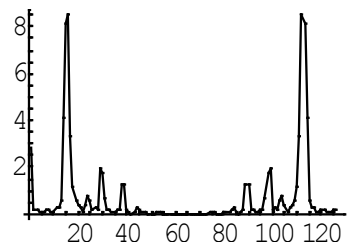

c)

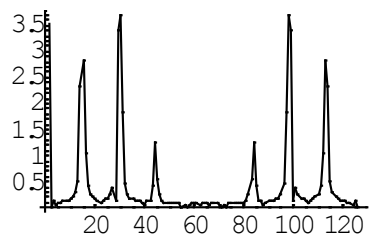

e)

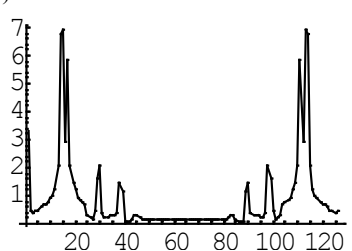

b)

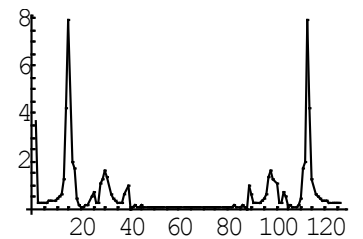

d)

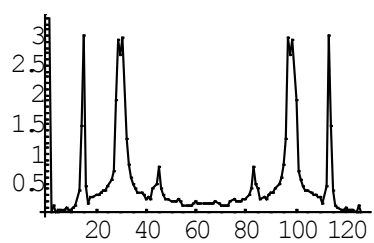

f)

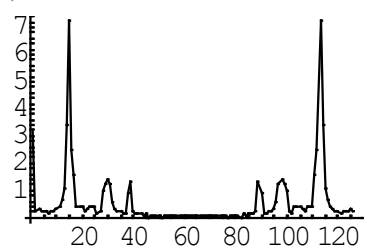

Figure 3. Spectra of the concentration oscillations corresponding to different successive steps of blood cell differentiation under in vitro conditions for healthy donors. The spectra demonstrate a reversible transition or FPU recurrence between basic complex attractors interpreted as different states of healthy cell differentiation. Fourier transforms of complex attractors (which have pedestals, unlike the lines of ordinary oscillations) correspond to (a, b) polymorphonuclear neutrophils and lymphocytes in the first and the second cycles, (c, d) lymphocytes and neutrophils in the middle of both cycles, and $(e, f)$ neutrophils and lymphocytes at the end of both cycles.

a)

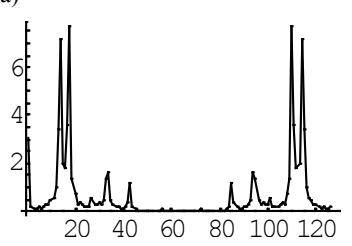

c)

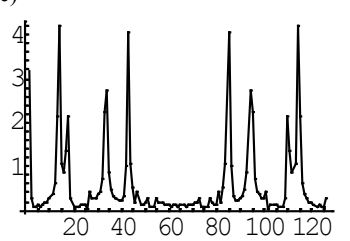

e)

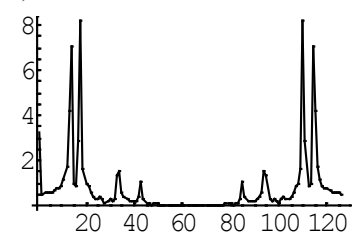

b)

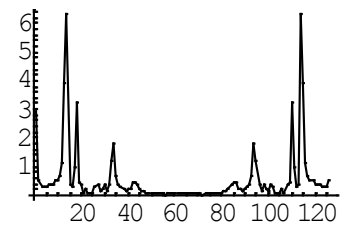

d)

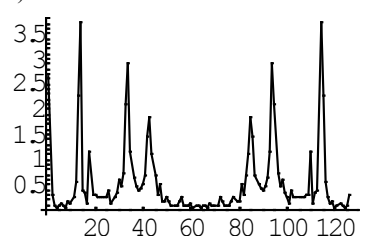

f)

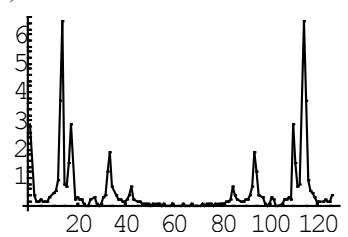

Figure 4. Spectra of the concentration oscillations corresponding to different successive steps of blood cell differentiation under in vitro conditions for acute-leukemia patients. The spectra demonstrate a reversible transition or FPU recurrence between basic (a, c, e) complex and (b, d, f) simple attractors interpreted as different states of the healthy (lymphocytes) and the leukemic (tumor) cell differentiation. Fourier transforms of simple attractors have a split pedestal (in contrast to a continuous pedestal for complex attractors) and correspond to (a,b) lymphocytes and tumor cells in the first and the second cycles, (c, d) neutrophils and tumor cells in the middle of both cycles, and (e, f) lymphocytes and tumor cells at the end of both cycles.

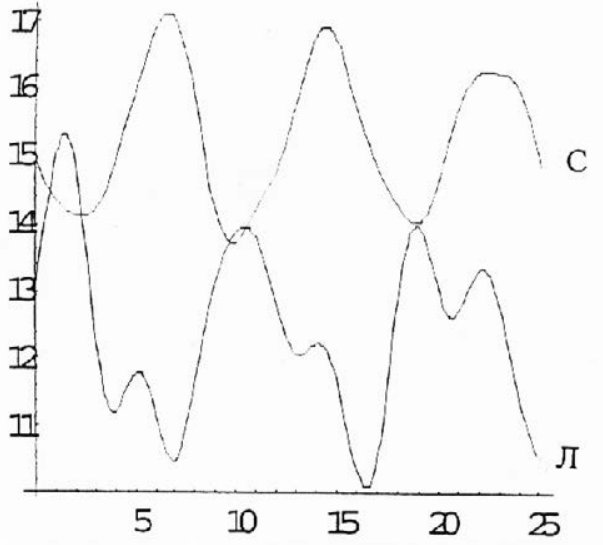

Figure 5. Simulated oscillations in concentrations of polymorphonuclear neutrophils (PN) and lymphocytes (L) in the in vitro blood of healthy donors in the framework of coupled discrete Ginzburg--Landau chains. The ordinate is the cell concentration .Units conditional.

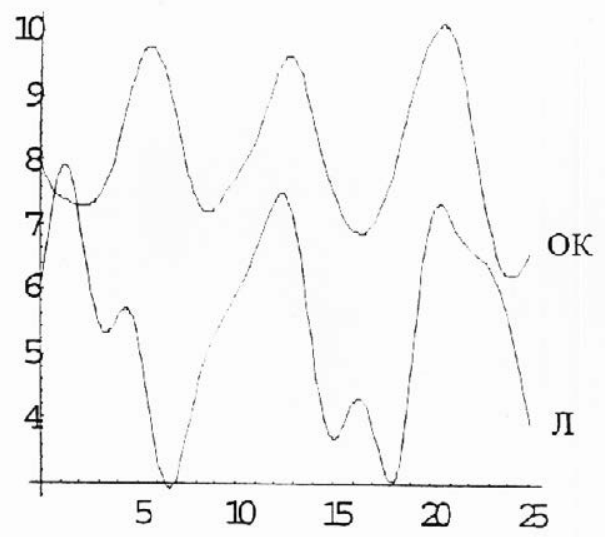

Figure 6. Simulated oscillations in concentrations of lymphocytes (L) and tumor cells (TC) in the $\{\mid \mathrm{em}$ in vitro $\}$ blood of acute-leukemia patients in the framework of coupled discrete Ginzburg--Landau chains. The ordinate is the cell concentration. Units conditional.

FPU recurrence occurs). The revealed property can be attributed to a hypothetical mechanism of activation of some genes in a DNA molecule and repression of the others. In the context of the results obtained in the first model [4], this mechanism represents a permanent external structuration of the blood cell differentiation attractors due to the introduction of the FPU recurrence spectra (occurring in the dynamics of mechanical oscillations in the DNA molecule) into these attractors. The number of possible scenarios of the dynamics of this system corresponds to the number of stable states of blood cell differentiation (or the number of attractors). Within the model of coupled Ginzburg-Landau chains, the development of leukemia is described as a decrease in the dimension of in vitro blood cell differentiation attractors. This decrease is due to genetic variations in the blood cells and brings about the transition from the cycle of complex attractors (or FPU recurrences) of differentiation states (i.e., healthy cells) to the cycle of simple attractors of differentiation states (describing the populations of tumor (leukemic) cells). As was shown in the first model [4], the reason for the decrease of the attractor dimension is the change in the vibrational parameters of the DNA molecule because of the violation of the molecular structure or molecular fragmentation (invasion of a virus, radiation injury, and incorporation of carcinogenic substances into the molecule). For the sake of simplicity, the effect of these changes in the DNA molecule was, in the model involving coupled Ginzburg- 
Berezin AA (2017) Fermi-Pasta-Ulam recurrence and coupled discrete Ginzburg-Landau chains in the description of various in vitro states of cell differentiation in the blood of healthy donors and acute-leukemia patients

-Landau equations, reduced to a decrease in the chain dimension (because these changes were simulated in the first model). The results of the simulation agreed qualitatively with the experimental data obtained.

Thus, the fundamental approach utilizing the Ginzburg-Landau chain and the FPU recurrence concept allowed us to describe the mechanism of transition of healthy blood cells into leukemic ones in terms of a decrease of the attractor dimension or the FPU recurrence complexity in a dynamical system. This is a qualitatively new result, which was not evident in the initial mathematical simulation of the experimental data [4].

\section{References}

1. Truman DE (1974) The Biochemistry of Citodifferentiation Blackwell Scientific Publications. Oxford, London, Edinburg, Melbourne.

2. Morgan TN (1934) Embryology and Genetics, New York Columbia University Press.
3. Spiegelman S (1948) Differentiation as the controlled production of unique enzymatic patterns. Symposia Soci Experim Biol 2: 286-325.

4. Berezin AA, Kratkie S (2002) Physical Approach to Simulation of Blood Cel Concentration Oscillations in Healthy and Leukemia Affected Organisms \{in vivo\} Bulletin of Lebedev's Institute of Physics 8: 34-44.

5. Gaponov-Grekhov AV, Rabinovich MI (1987) Avtostruktury Khaoticheskaya dinamika ansamblei (Autostructures and chaotic dynamics of ensembles), in Nelineinye volny (Nonlinear Waves), Moscow, Nauka, Pp. 123.

6. Fermi E, Pasta J, Ulam S (1955) In the Collected papers of Enrico Fermi. 2: 978.

7. Yudovich VI (1977) Asimptotika predel'nykh tsiklov sistemy Lorentsa pri bol'shikh chislakh Releya (Asymptotics of Limit Cycles of the Lorentz System for Large Rayleigh Numbers), Rostov-on-Don, Rostov State University.

8. Lorenz E (2002) Deterministic Nonperiodic Flow. J Atmos Sci 20: 130.

9. Makarov MS (1975) Rol' granulotsitov v protsesse vospalitel'noi regeneratsii po dannym sravnitel'nogo patologicheskogo issledovaniya (The Role of Granulocytes in the Process of Inflammatory Regeneration on Evidence Derived from Comparative Cytological Study), Stavropol', Izd. Kubanskogo meditsinskogo instituta (Kuban Medical Institute)

Copyright: $@ 2017$ Berezin AA. This is an open-access article distributed under the terms of the Creative Commons Attribution License, which permits unrestricted use, distribution, and reproduction in any medium, provided the original author and source are credited. 